\title{
Development of a multivariable prognostic PREdiction model for 1-year risk of FALLing in a cohort of community-dwelling older adults aged 75 years and above (PREFALL)
}

Gustav Valentin Gade ${ }^{1,2^{*}}$ (D), Martin G. Jørgensen ${ }^{1}$ (D) Jesper Ryg ${ }^{3,4}$ (D), Tahir Masud ${ }^{3,5}$ (D) Lasse Hjort Jakobsen ${ }^{2,6}$ and Stig Andersen ${ }^{1,2}$ (ID

\begin{abstract}
Background: Falls are the leading cause of fatal and non-fatal injuries in older adults, and attention to falls prevention is imperative. Prognostic models identifying high-risk individuals could guide fall-preventive interventions in the rapidly growing older population. We aimed to develop a prognostic prediction model on falls rate in community-dwelling older adults.

Methods: Design: prospective cohort study with 12 months follow-up and participants recruited from June 14, 2018, to July 18, 2019.

Setting: general population.

Subjects: community-dwelling older adults aged 75+ years, without dementia or acute illness, and able to stand unsupported for one minute.

Outcome: fall rate for 12 months.

Statistical methods: candidate predictors were physical and cognitive tests along with self-report questionnaires. We developed a Poisson model using least absolute shrinkage and selection operator penalization, leave-one-out crossvalidation, and bootstrap resampling with 1000 iterations.

Results: Sample size at study start and end was 241 and 198 (82\%), respectively. The number of fallers was 87 (36\%), and the fall rate was 0.94 falls per person-year. Predictors included in the final model were educational level, dizziness, alcohol consumption, prior falls, self-perceived falls risk, disability, and depressive symptoms. Mean absolute error $(95 \% \mathrm{Cl})$ was 0.88 falls $(0.71-1.16)$.
\end{abstract}

\footnotetext{
* Correspondence: gustavs@dcm.aau.dk

'Department of Geriatric Medicine, Aalborg University Hospital, Hobrovej 18-22, Gl. Roed Bygn. 6, 9000 Aalborg, Denmark

${ }^{2}$ Department of Clinical Medicine, Aalborg University, Soendre Skovvej 15 9000 Aalborg, Denmark

Full list of author information is available at the end of the article
}

C The Author(s). 2021 Open Access This article is licensed under a Creative Commons Attribution 4.0 International License, which permits use, sharing, adaptation, distribution and reproduction in any medium or format, as long as you give appropriate credit to the original author(s) and the source, provide a link to the Creative Commons licence, and indicate if changes were made. The images or other third party material in this article are included in the article's Creative Commons licence, unless indicated otherwise in a credit line to the material. If material is not included in the article's Creative Commons licence and your intended use is not permitted by statutory regulation or exceeds the permitted use, you will need to obtain permission directly from the copyright holder. To view a copy of this licence, visit http://creativecommons.org/licenses/by/4.0/. The Creative Commons Public Domain Dedication waiver (http://creativecommons.org/publicdomain/zero/1.0/) applies to the data made available in this article, unless otherwise stated in a credit line to the data. 
Conclusion: We developed a falls prediction model for community-dwelling older adults in a general population setting. The model was developed by selecting predictors from among physical and cognitive tests along with selfreport questionnaires. The final model included only the questionnaire-based predictors, and its predictions had an average imprecision of less than one fall, thereby making it appropriate for clinical practice. Future external validation is needed.

Trial registration: Clinicaltrials.gov (NCT03608709).

Keywords: Accidental falls, Models, Theoretical, Multivariable analysis, Prognosis

\section{Background}

Fall accidents are frequent in community-dwelling older adults. Around $30 \%$ of all $65+-$ year olds fall yearly. This increases to $50 \%$ in older adults aged 80 years or above [1]. With the current demographic trends, the proportion of older adults will increase [1], and consequently, the number of people falling will rise. Falls are associated with increased morbidity and mortality along with loss of independence, which ultimately may lead to earlier placement in long-term care [2-4]. Furthermore, health care costs related to falls increase with age [5]. Therefore, early falls prevention is imperative and may be most beneficial to individuals at high risk of falling [6].

Identifying high-risk individuals is not straightforward due to the multifactorial and complex nature of falls with more than 400 acknowledged risk factors [4, 7]. Furthermore, these associations refer to group differences on specific risk factors. In contrast, personal predictions are estimates of absolute risks on an individual level [8]. Prognostic models predict an individual's risk of a future outcome and are typically developed by evaluating two or more risk factors in combination [9]. Thus, older adults may have their risk of falling estimated to better target interventions. A recently published systematic review [10] highlighted models available for predicting falls [11-17]. These models were primarily based on younger participants (65+ years) [1113, 15-17]. Also, methodological limitations were present regarding outcome assessments with long recording intervals and lack of blinding [11-17] which could potentially lead to unreliable predictions. Moreover, to support the implementation of models, applicability in clinical practice is a vital issue to address [18].

This study aimed to develop a multifactorial prognostic prediction model for estimating the risk of falling in community-dwelling older adults.

\section{Methods}

This prospective cohort study recruited participants from $14 / 06 / 2018$ to $18 / 07 / 2019$, with a 12-month follow-up period. A pre-registered protocol was submitted at Clinicaltrials.gov (NCT03608709) [19] on 01/08/ 2018. The reporting of the study followed the
Transparent Reporting of a multivariable prediction model for Individual Prognosis Or Diagnosis (TRIPOD) statement [20] and Strengthening the Reporting of OBservational studies in Epidemiology (STROBE) statement [21]. TRIPOD and STROBE checklists are available in Additional file 1: appendix 1 . To adhere fully to the reporting guidelines, a more detailed description of the population, recruitment sites, study dates, data collection procedures on predictors and outcomes, along with statistical analyses and results are available in Additional file 1: appendix 2.

\section{Sources of data}

This was a study conducted in a general population setting within a single municipality.

\section{Participants}

Community-dwellers aged 75 years or above residing in Hjørring Municipality, Northern Jutland, Denmark, were studied. We recruited participants through preventive home visits (PHV), senior activity centers (SAC), along with senior clubs and associations. Participants were assessed for eligibility and included consecutively in PHVs and by convenience in the remaining recruitment sites. Exclusion criteria were living in care facilities, the presence of self-reported acute illness within seven days before recruitment, being unable to stand for one minute without any assistive device or support from another person, unable to understand Danish, or having a dementia diagnosis. The latter was checked through hospital records with informed consent from participants. Data collectors only recorded whether the older adults were eligible for participation or not due to time constraints.

\section{Outcome}

We defined falls as "an unexpected event in which the participants come to rest on the ground floor or lower level" [22]. The primary outcome for prediction was falls rate within 12 months. Fall calendars with a daily recording of falls were returned monthly by post. If calendars had falls recorded, research secretaries contacted participants by telephone to validate and describe the falls. 


\section{Predictors}

The test battery has been described previously [23]. In brief, it consisted of physical tests of static balance under dual-tasking conditions, grip- and lower limb strength, reaction time of lower limbs, and habitual gait speed; a self-report questionnaire on demographic characteristics, frailty, nutrition, disability, fear of falling, health-related quality of life, depression, several physical symptoms, and a cognitive test. Static balance, reaction time, gripand lower limb strength were measured using a Nintendo Wii Balance Board due to its valid and reliable measures and portable setup [24-27]. Gait speed was measured using the four-meter gait speed test [28]. Furthermore, frailty was assessed using Tilburg Frailty Indicator (TFI) [29], nutrition using the short Mini Nutritional Assessment (MNA) [30], disability using Vulnerable Elders Survey 13, (VES) [31], fear of falling using short Falls Efficacy Scale International [32], health-related quality of life using EuroQol Five Dimensions Three Levels (EQ-5D-3L), depressive symptoms using the Geriatric Depression Scale with four items (GDS) [33], and the cognitive test using the OrientationMemory-Concentration test [34]. In total, 34 candidate predictors corresponding to 41 degrees of freedom were available for model building. Additional file 1: Appendix 3. eTable 1 provides details of all candidate predictors, how they were defined, along with how and when they were measured.

\section{Blinding}

Municipality staff and the first author performed the baseline data collection. Data collectors in PHVs and SACs were blinded to all predictors except the physical tests. Participants were blinded to all predictors except those in the questionnaire and the number of all prescribed drugs. The first author was blinded to physical tests performed in PHVs and SACs. Research secretaries assessing the outcome were blinded to baseline predictors.

\section{Statistical analyses}

A recommended method for calculating sample size was not available when the study commenced. Thus, we based the sample size on feasible recruitment within the 13 months inclusion period granted by the municipality.

\section{Descriptive statistics and comparisons}

We summarized continuous variables using medians with interquartile range. Categorical variables were summarized using proportions. We compared baseline demographics between recruitment sites and follow-up completeness (Additional file 1: appendix 2). Finally, we calculated univariate falls incidence rate ratios (IRR) for all predictors using Poisson regression.

\section{Missing data}

We summed the number of missing values per predictor and the number of subjects with missing data. We compared participants with and without missing data similarly to follow-up completeness comparison (see Additional file 1: appendix 2). Missing data were imputed by a single imputation in a random forest imputation scheme to avoid excessive computation time associated with multiple imputation techniques and preserve imputations' precision [35]. However, we chose a mode imputation scheme for categorical variables with less than ten observations in a category. The imputation procedures used all candidate predictors without missing data (see Additonal file 1: appendix 3 - eTable 1).

\section{Handling of predictors in the analyses}

All continuous variables were kept continuous and modeled linearly to avoid loss of information by dichotomization [36]. Variables used for generating a score, e.g., the Tilburg Frailty Indicator, were not included in the modeling process; we only included the scores. Scores were generated after imputing missing data. For categorical variables, all cut points were pre-specified and summarized in Additional file 1: appendix 3. eTable 1.

\section{Modeling}

Follow-up time was defined as the time from inclusion until death, loss to follow-up, or end of the study. Fall rate was calculated as the total number of falls divided by length of follow-up in years. The fall rate was modeled using a Poisson regression model with log-followup time as off-set and number of falls as the dependent variable. We fitted the model using least absolute shrinkage and selection operator penalization [37] with leaveone-out cross-validation to perform variables selection and address overfitting during modeling. Thus, the abovementioned univariate analyses did not guide predictor selection in the modeling process. Interaction terms were not tested due to limited sample size. Furthermore, to guide the prediction modeling process, we pre-specified the expected direction of predictor effects (Additional file 1: appendix 3 - eTable 1). Performance measures were internally validated using a bootstrap resampling technique with 1000 samples drawn with replacement from the original sample. The model was fitted on the bootstrap sample and validated on the outof-bag sample. Participants who died or were lost to follow-up were always included in the bootstrap sample as the true number of falls was not observed in these participants. To quantify model performance, we computed the mean absolute difference between each participant's predicted and observed number of falls. This is also known as the mean absolute error (MAE) and reflects how imprecisely the model predicts on the entire 
sample using the unit: number of falls. We derived a 95\% confidence interval from the 2.5th and 97.5th percentiles of the 1000 MAEs from the bootstrap resampling procedure. Lastly, we also calculated the mean squared error (MSE) with a 95\% confidence interval.

\section{Software}

All statistical analyses were performed using $\mathrm{R}$, version 4.0.2, ( $\mathrm{R}$ Foundation) with packages: $\mathrm{mlr}$, glmnet, pseudo, pROC, and reshape2.

\section{Results}

\section{Descriptive characteristics}

During the study period, 6197 community-dwelling older adults (75+ years) lived in the municipality. Of these, we assessed 912 for eligibility, from which we included 241 older adults (66.4\% women) with median (IQR) age $82(80 ; 86)$ years. Figure 1 displays the flow of participants, and Table 1 provides the baseline characteristics of participants. Fifteen (6\%) participants had missing data on predictors. Their demographic characteristics did not differ from participants without missing data.

\section{Outcome}

The median (IQR) follow-up time for all participants was 365 (365-365) days. Forty-three (18\%) participants had an incomplete follow-up. Median (IQR) age was higher for these, 85 (81-87) years, compared to participants retained in the study, $82(79-86)$ years, $(p=0.01)$. Eighty-seven (36\%) participants fell during follow-up, and 44 of these fell more than once. In total, we recorded 178 falls and calculated a fall rate of 0.94 falls per person-year. Table 2 displays univariate falls IRR for all predictors. Risk factors associated with falls were the completion of a short-cycle or medium-cycle higher education (IRR 2.97, 95\% CI 1.80-4.74; IRR 2.00, 95\% CI 1.36-2.92), drinking more than seven units of alcohol

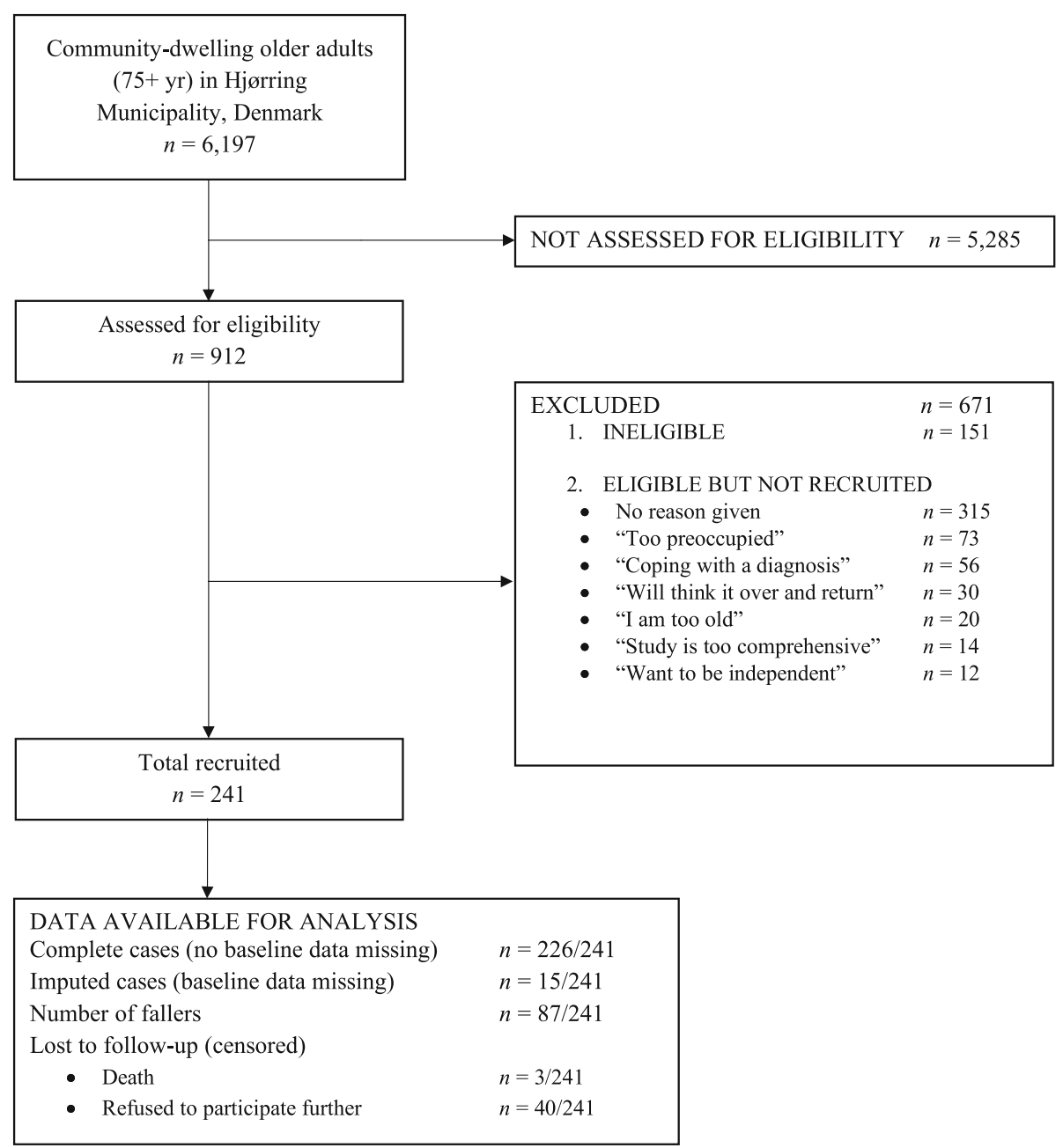

Fig. 1 Flow diagram displaying participants at each stage in the study 
Table 1 Cohort baseline characteristics

\begin{tabular}{|c|c|c|}
\hline Variable & Level and summary measure & Total $^{\text {a }}$ \\
\hline \multicolumn{3}{|l|}{ Sociodemographics } \\
\hline Age (years) & median $[\mathrm{IQR}]$ & $82[80,86]$ \\
\hline Sex & Women, n (\%) & $160(66.4)$ \\
\hline \multirow[t]{3}{*}{ Marital status } & Married or living with a partner, n (\%) & $98(40.7)$ \\
\hline & Widow, n (\%) & $125(51.9)$ \\
\hline & Other, n (\%) & $18(7.5)$ \\
\hline Living alone & Yes, n (\%) & $144(59.8)$ \\
\hline \multirow[t]{3}{*}{ Highest level of education completed } & Municipal primary and lower secondary school, n (\%) & $106(44.0)$ \\
\hline & Skilled worker, n (\%) & $68(28.2)$ \\
\hline & General upper secondary education or longer, n (\%) & $67(27.8)$ \\
\hline \multirow[t]{3}{*}{ Monthly household income in Euro } & $\leq 1075, \mathrm{n}(\%)$ & $106(44.0)$ \\
\hline & $1076-2285, \mathrm{n}(\%)$ & $81(33.6)$ \\
\hline & $>2285, \mathrm{n}(\%)$ & $54(22.4)$ \\
\hline \multirow[t]{3}{*}{ Weekly alcohol consumption (units) } & $<7, \mathrm{n}(\%)$ & $187(77.6)$ \\
\hline & $7-14, \mathrm{n}(\%)$ & $47(19.5)$ \\
\hline & $>14, \mathrm{n}(\%)$ & $7(2.9)$ \\
\hline Having dog or cats in the household & Yes, n (\%) & $30(12.4)$ \\
\hline Using glasses with multifocal lenses & Yes, n (\%) & $184(76.3)$ \\
\hline \multicolumn{3}{|l|}{ Falls } \\
\hline One or more falls within the previous year & Yes, n (\%) & $105(43.6)$ \\
\hline Do you think you will fall within the next year? & Yes, n (\%) & $45(18.7)$ \\
\hline Short Falls Efficacy Scale International (7-28) \# & median $[\mathrm{IQR}]$ & $8[7,11]$ \\
\hline \multicolumn{3}{|l|}{ Comorbidities and medication } \\
\hline Having two or more diseases or chronic disorders & Yes, n (\%) & $209(86.7)$ \\
\hline Charlson Comorbidity Index - score (0-37) \# & median [IQR] & $1[0,2]$ \\
\hline \multirow[t]{3}{*}{ Charlson Comorbidity Index - grouping \# } & $0, n(\%)$ & $106(44.0)$ \\
\hline & $1-2, \mathrm{n}(\%)$ & $108(44.8)$ \\
\hline & $\geq 3, \mathrm{n}(\%)$ & $27(11.2)$ \\
\hline Number of all prescribed drugs & median [IQR] & $7[4,10]$ \\
\hline Polypharmacy (5 or more prescribed drugs) & Yes, n (\%) & $170(70.5)$ \\
\hline \multicolumn{3}{|l|}{ Frailty and symptoms } \\
\hline Tilburg Frailty Indicator - Total score (0-15) \# & median [IQR] & $6[4,8]^{b}$ \\
\hline Occasionally experiencing dizziness & Yes, n (\%) & $123(51.0)$ \\
\hline Occasionally experiencing lower limb pain when walking? & Yes, n (\%) & $121(50.2)$ \\
\hline Occasionally experiencing urinary incontinence & Yes, n (\%) & $90(37.3)$ \\
\hline \multicolumn{3}{|l|}{ Nutrition } \\
\hline Mini Nutritional Assessment Score (0-14) & median [IQR] & $11[10,12]^{b}$ \\
\hline Body Mass Index (kg/m²) & median $[\mathrm{IQR}]$ & $27.7[25.3,30.7]^{\mathrm{b}}$ \\
\hline \multicolumn{3}{|l|}{ Psychology and cognition } \\
\hline Geriatric Depression Scale - 4 items \# & Two or more points, $\mathrm{n}(\%)$ & $13(5.4)$ \\
\hline Orientation-Memory-Concentration test (0-28) & median [IQR] & $26[22,26]^{d}$ \\
\hline EQ-5D-3L score $(-0.167-1.000)$ & median [IQR] & $0.7[0.6,1.0]$ \\
\hline \multicolumn{3}{|l|}{ Activities of Daily Living and mobility } \\
\hline Vulnerable Elders Survey - $13(1-10)$ \# & median $[\mathrm{IQR}]$ & $3[1,4]^{d}$ \\
\hline
\end{tabular}


Table 1 Cohort baseline characteristics (Continued)

\begin{tabular}{|c|c|c|}
\hline Variable & Level and summary measure & Total $^{\text {a }}$ \\
\hline Using walking aids & Yes, n (\%) & $142(58.9)$ \\
\hline Habitual gait speed (m/sec) & median [IQR] & $0.9[0.7,1.2]$ \\
\hline \multicolumn{3}{|l|}{ Physical } \\
\hline Total grip strength of both upper limbs (kg) & median $[\mathrm{IQR}]$ & $24.3[18.6,30.2]^{c}$ \\
\hline Difference in grip strength between upper limbs (kg) & median $[\mathrm{IQR}]$ & $1.5[0.7,2.5]^{c}$ \\
\hline Total isometric lower limb strength (kg) & median [IQR] & $132.8[90.1,188.4]^{c}$ \\
\hline Difference in isometric lower limb strengths (kg) & median [IQR] & $7.1[3.4,11.9]^{\mathrm{c}}$ \\
\hline Average lower limb reaction time (ms) & median [IQR] & $1315[1122 ; 1567]^{c}$ \\
\hline Difference in lower limb reaction time (ms) & median $[\mathrm{IQR}]$ & $142[60,370]^{c}$ \\
\hline Centre of Pressure area $\left(\mathrm{mm}^{2}\right)$ & median $[\mathrm{IQR}]$ & $69[42,112]^{b}$ \\
\hline Centre of Pressure speed $(\mathrm{mm} / \mathrm{s})$ & median $[\mathrm{IQR}]$ & $23[16,30]^{b}$ \\
\hline
\end{tabular}

Notes: $n$ number, \% percentage proportion, $I Q R$ interquartile range, \# a lower score is better, $\mathrm{m} / \mathrm{sec}$ meters per second, $\mathrm{kg}$ kilogram, $\mathrm{ms}$ milliseconds, $\mathrm{mm}{ }^{2}$ square millimeters, $\mathrm{mm} / \mathrm{s}$ millimeters per second, $\mathrm{kg} / \mathrm{m}^{2} \mathrm{~kg}$ per square meters, ${ }^{a}=$ sample size is 241 participants, ${ }^{\mathrm{b}}=1 \mathrm{missing}$ observation, ${ }^{\mathrm{c}}=2 \mathrm{missing}$ observations, ${ }^{\mathrm{d}}=$ 4 missing observations

weekly (IRR 1.55, 95\% CI 1.12-2.11), using walking aids (IRR 1.37, 95\% CI 1.02-1.83), having three points on the Geriatric Depression Scale (IRR 9.5, 95\% CI 5.34-15.70), having fallen within the last year (IRR 3.47, 95\% CI 2.53-4.85), believing to fall within the next year (IRR 4.10, 95\% CI 3.04-5.50), presence of dizziness (IRR 2.17, 95\% CI 1.59-2.99), and increased balance sway area when dual-tasking (IRR 1.00, 95\% CI 1.00-1.00). An increasing number of prescribed drugs (IRR 1.06, 95\% CI 1.02-1.10), TFI score (IRR 1.13, 95\% CI 1.06-1.20), Short Falls Efficacy Scale score (IRR 1.06, 95\% CI 1.011.11), and VES score (IRR 1.13, 95\% CI 1.07-1.18) were also associated with falls. Finally, use of multifocal lenses (IRR 0.60, 95\% CI 0.44-0.83) along with increasing age (IRR 0.97, 95\% CI 0.94-1.00), MNA score (IRR 0.84, 95\% CI 0.78-0.91), and EQ-5D-3L score (IRR 0.19, 95\% CI 0.09-0.40) were associated with a lower falls rate.

\section{Prediction modeling}

Figure 2 displays which predictors were most frequently selected for the prediction model in each bootstrap. Table 3 gives the fully specified prediction model and its performance measures. The final model included educational level, dizziness, weekly alcohol consumption, prior falls, self-perceived falls risk, disability, and depressive symptoms; all reported in a 22 -item self-report questionnaire. For apparent performance, i.e., before internal validation, the MAE was 0.80 falls. After internal validation, MAE (95\% CI) increased to $0.88(0.71-1.16)$ falls. Additional file 1: Appendix 4. eTable 2 gives an example of how to use the model.

\section{Discussion}

This study aimed to develop a falls prediction model for community-dwelling older adults. Only self-reported predictors were included in the final model. This makes it a simple and clinically applicable model. The model predictions had an average imprecision of less than one fall.

\section{Comparison to other studies}

Other studies have developed falls prediction models for community-dwelling older adults with the same setting and falls definition as in the present study [11-17]. Models were either purely questionnaire-based [13, 15, 17] or a combination of questionnaires and objective measures $[11,12,15,16]$. Similarly, they found prior falls [12, 13, 15-17], self-perceived falls risk [17], activities of daily living $[12,16]$, dizziness $[12,16]$, educational level $[12,16]$, alcohol consumption $[12,16]$, and depressive symptoms [11] to predict falls. In contrast to these studies, we did not find self-reported visual impairment [11, $13,15]$, grip strength $[12,16]$, body mass index $[11,12]$, medication [13], or global cognition [11] to be predictive of falls. This discrepancy may be due to different participant characteristics along with assessment methods for outcomes and predictors. For participant characteristics, younger participants ( $\geq 65$ years) were included [11-13, 15-17] resulting in a median age around 75 years for the majority of studies $[11-13,16]$. In contrast, our sample was older but smaller. Thus, our composition of predictors may only be representative for older adults (75+ years). For outcome assessments, we applied fall calendars, with a daily recording of falls, returned monthly, and outcome assessors blinded to baseline predictors. For the other studies, falls were recorded either yearly $[15]$, quarterly $[13,16,17]$, or weekly $[11,12,14]$ which may increase the risk of recall bias and influence predictor selection for models. Also, only one study reported whether outcome assessors were blinded when 
Table 2 Univariate falls incidence rate ratios for characteristics of 241 community-dwelling older adults (75+ years)

\begin{tabular}{|c|c|c|c|}
\hline Variable & Unit or category & IRR $(95 \% \mathrm{Cl})$ & $p$-value \\
\hline \multicolumn{4}{|l|}{ Sociodemographics } \\
\hline Age (years) & Years & $0.97(0.94-1.00)$ & $<.05$ \\
\hline \multirow[t]{2}{*}{ Sex } & Men & Ref & Ref \\
\hline & Women & $0.90(0.67-1.23)$ & .52 \\
\hline \multirow[t]{4}{*}{ Marital status } & Married or living with a partner & Ref & Ref \\
\hline & Not married & $1.44(0.60-2.90)$ & .36 \\
\hline & Separated or divorced & $0.91(0.35-1.93)$ & .83 \\
\hline & Widow & $1.08(0.79-1.47)$ & .64 \\
\hline \multirow[t]{2}{*}{ Living alone } & No & Ref & Ref \\
\hline & Yes & $1.19(0.88-1.61)$ & .27 \\
\hline \multirow[t]{6}{*}{ Highest level of education completed } & Municipal primary and lower secondary school & Ref & Ref \\
\hline & General upper secondary education & $0.76(0.19-0.21)$ & .64 \\
\hline & Skilled worker & $1.01(0.67-1.49)$ & .97 \\
\hline & Short-cycle higher education & $2.97(1.80-4.74)$ & $<.05$ \\
\hline & Medium-cycle higher education & $2.00(1.36-2.92)$ & $<.05$ \\
\hline & Long cycle higher education & $0.84(0.25-2.03)$ & .73 \\
\hline \multirow[t]{2}{*}{ Weekly alcohol consumption } & $0-7$ units & Ref & Ref \\
\hline & $>7$ units & $1.55(1.12-2.11)$ & $<.05$ \\
\hline \multirow[t]{2}{*}{ Dogs or cats in the household } & No & Ref & Ref \\
\hline & Yes & $1.26(0.82-1.85)$ & .27 \\
\hline \multirow[t]{2}{*}{ Using glasses with multifocal lenses } & No & Ref & Ref \\
\hline & Yes & $0.60(0.44-0.83)$ & $<.05$ \\
\hline \multicolumn{4}{|l|}{ Comorbidities and medication } \\
\hline \multirow[t]{2}{*}{ Having two or more diseases or chronic disorders } & No & Ref & Ref \\
\hline & Yes & $1.35(0.86-2.25)$ & .21 \\
\hline Number of all prescribed drugs & Count & $1.06(1.02-1.10)$ & $<.05$ \\
\hline \multicolumn{4}{|l|}{ Nutrition } \\
\hline Body Mass Index & $\mathrm{Kg} / \mathrm{m}^{2}$ & $0.98(0.95-1.01)^{a}$ & .24 \\
\hline Mini Nutritional Assessment Score & Score from 0 to 14 (14 is the best possible score) & $0.84(0.78-0.91)^{\mathrm{a}}$ & $<.05$ \\
\hline \multicolumn{4}{|l|}{ Activities of Daily Living and mobility } \\
\hline Vulnerable Elders Survey - 13 & Score from 1 to 10 ( 1 is the best score) & $1.13(1.07-1.18)$ & $<.05$ \\
\hline \multirow[t]{2}{*}{ Using walking aids } & No & Ref & Ref \\
\hline & Yes & $1.37(1.02-1.83)$ & $<.05$ \\
\hline Habitual gait speed & $\mathrm{M} / \mathrm{sec}$ & $0.88(0.65-1.18)$ & .41 \\
\hline \multicolumn{4}{|l|}{ Psychology and cognition } \\
\hline \multirow[t]{4}{*}{ Geriatric Depression Scale - 4 items } & 0 & Ref & Ref \\
\hline & 1 & $0.76(0.50-1.13)$ & .20 \\
\hline & 2 & $1.15(0.54-2.14)$ & .67 \\
\hline & 3 & $9.5(5.34-15.70)$ & $<.05$ \\
\hline Orientation-Memory-Concentration test & Score from 0 to 28 (28 is the best score) & $0.99(0.96-1.03)^{c}$ & .69 \\
\hline$E Q-5 D-3 L$ & Score from -0.167 to 1.000 points & $0.19(0.09-0.40)$ & $<.05$ \\
\hline \multicolumn{4}{|l|}{ Falls } \\
\hline \multirow[t]{2}{*}{ One or more falls within the previous year } & No & Ref & Ref \\
\hline & Yes & $3.47(2.53-4.85)$ & $<.05$ \\
\hline
\end{tabular}


Table 2 Univariate falls incidence rate ratios for characteristics of 241 community-dwelling older adults (75+ years) (Continued)

\begin{tabular}{|c|c|c|c|}
\hline Variable & Unit or category & IRR $(95 \% \mathrm{Cl})$ & $p$-value \\
\hline Short Falls Efficacy Scale International & Score from 7 to 28 ( 7 is the best score) & $1.06(1.01-1.11)$ & $<.05$ \\
\hline \multirow[t]{2}{*}{ Do you think that you will fall within the next year? } & No & Ref & Ref \\
\hline & Yes & $4.10(3.04-5.50)^{b}$ & $<.05$ \\
\hline \multicolumn{4}{|l|}{ Frailty and symptoms } \\
\hline Tilburg Frailty Indicator & Score from 0 to 15 points ( 0 is the best possible score) & $1.13(1.06-1.20)^{a}$ & $<.05$ \\
\hline \multirow[t]{2}{*}{ Occasionally experiencing dizziness } & No & Ref & Ref \\
\hline & Yes & $2.17(1.59-2.99)$ & $<.05$ \\
\hline \multirow[t]{2}{*}{ Occasionally experiencing lower limb pain when walking? } & No & Ref & Ref \\
\hline & Yes & $1.29(0.96-1.74)$ & .09 \\
\hline \multirow[t]{2}{*}{ Occasionally experiencing urinary incontinence } & No & Ref & Ref \\
\hline & Yes & $1.25(0.92-1.67)$ & .15 \\
\hline \multicolumn{4}{|l|}{ Physical } \\
\hline Total grip strength of both upper limbs & $\mathrm{Kg}$ & $0.99(0.97-1.00)^{b}$ & .17 \\
\hline Difference in grip strength between upper limbs & $\mathrm{Kg}$ & $0.95(0.86-1.04)^{b}$ & .34 \\
\hline Total isometric lower limb strength & $\mathrm{Kg}$ & $0.98(0.96-1.00)^{b, d}$ & .08 \\
\hline Difference in isometric lower limb strengths & $\mathrm{Kg}$ & $0.78(0.62-0.97)^{b, d}$ & .03 \\
\hline Average lower limb reaction time & Ms & $0.99(0.99-1.00)^{b, d}$ & .96 \\
\hline Difference in lower limb reaction time & Ms & $0.99(0.99-1.00)$ b, e & .09 \\
\hline Centre of Pressure area & $\mathrm{mm}^{2}$ & $1.01(1.00-1.02)^{a, d}$ & $<.05$ \\
\hline Centre of Pressure speed & $\mathrm{mm} / \mathrm{s}$ & $1.04(0.94-1.14)^{a, d}$ & .45 \\
\hline
\end{tabular}

Notes: $n$ number, \% percentage proportion, IRR incidence rate ratio, $\mathrm{Cl}$ confidence interval, Ref reference group, $\mathrm{m} / \mathrm{sec}$ meters per second, $\mathrm{kg}$ kilogram, $\mathrm{ms}$ milliseconds, $\mathrm{mm}^{2}$ square millimeters, $\mathrm{mm} / \mathrm{s}$ millimeters per second, $\mathrm{kg} / \mathrm{m}^{2} \mathrm{~kg}$ per square meters, ${ }^{a}=1$ missing observation, ${ }^{b}=2$ missing observations, ${ }^{\mathrm{c}}=4$ missing observations, ${ }^{d}=$ data has been scaled by a factor of $10,{ }^{e}=$ IRR $(95 \% \mathrm{Cl}): 0.9997914(0.9995242-1.000003)$

validating falls according to their definition [17]. Thus, it is unclear whether detection bias influencing the predictive abilities of risk factors was present in the remaining studies. Lastly, differing measurement methods may cause different predictive abilities for the same risk factor across studies. These limitations can potentially lead to the inclusion of risk factors with poor predictive abilities in models. Consequently, model predictors may differ, and predictions may be less reliable when using models in clinical practice.

\section{Implications for clinical practice}

On a group level, we found 16 risk factors associated with falls through univariate analyses. However, only half of these were able to predict falls on an individual level. This emphasizes the need for clinicians to know when to best apply evidence from association and prediction modeling studies on falls since a statistically significant association does not imply that a risk factor is a strong predictor. For example, lower limb strength is strongly associated with falls [38] but may be less predictive of falls in community-dwelling older adults in the general population $[12,15,16]$. In our study, lower limb strength was not included in our prediction model, indicating no additional predictive value in the presence of the other candidate predictors. This was substantiated during our model development, where lower limb strength was less frequently chosen than the predictors in the final model. This finding is in line with three other prediction modeling studies measuring lower limb strength $[12,15,16]$. Here, only one study included a measure of lower limb strength in a final model but concluded that this was not superior to simpler questionnaire-based models [15]. Reasons for this could be because the test does not differentiate well between fallers and non-fallers as the average lower limb strength compares to the background population [39], and more functional tests of lower limb strength could be more relevant in terms of investigating for predictive performance. This knowledge may be applied when following the current recommendations for falls prevention [6]. Here, initial screening of falls risk is necessary for referring high-risk individuals to further assessment for modifiable risk factors and targeted interventions. Falls prediction models are appropriate for screening but not necessarily explaining falls risk. When presumed high-risk individuals have been identified, evidence on associated modifiable falls risk factors can explain why the older person is at risk and what to intervene on. In this scenario, self-report questionnaire models that predict falls may be both time-efficient and 


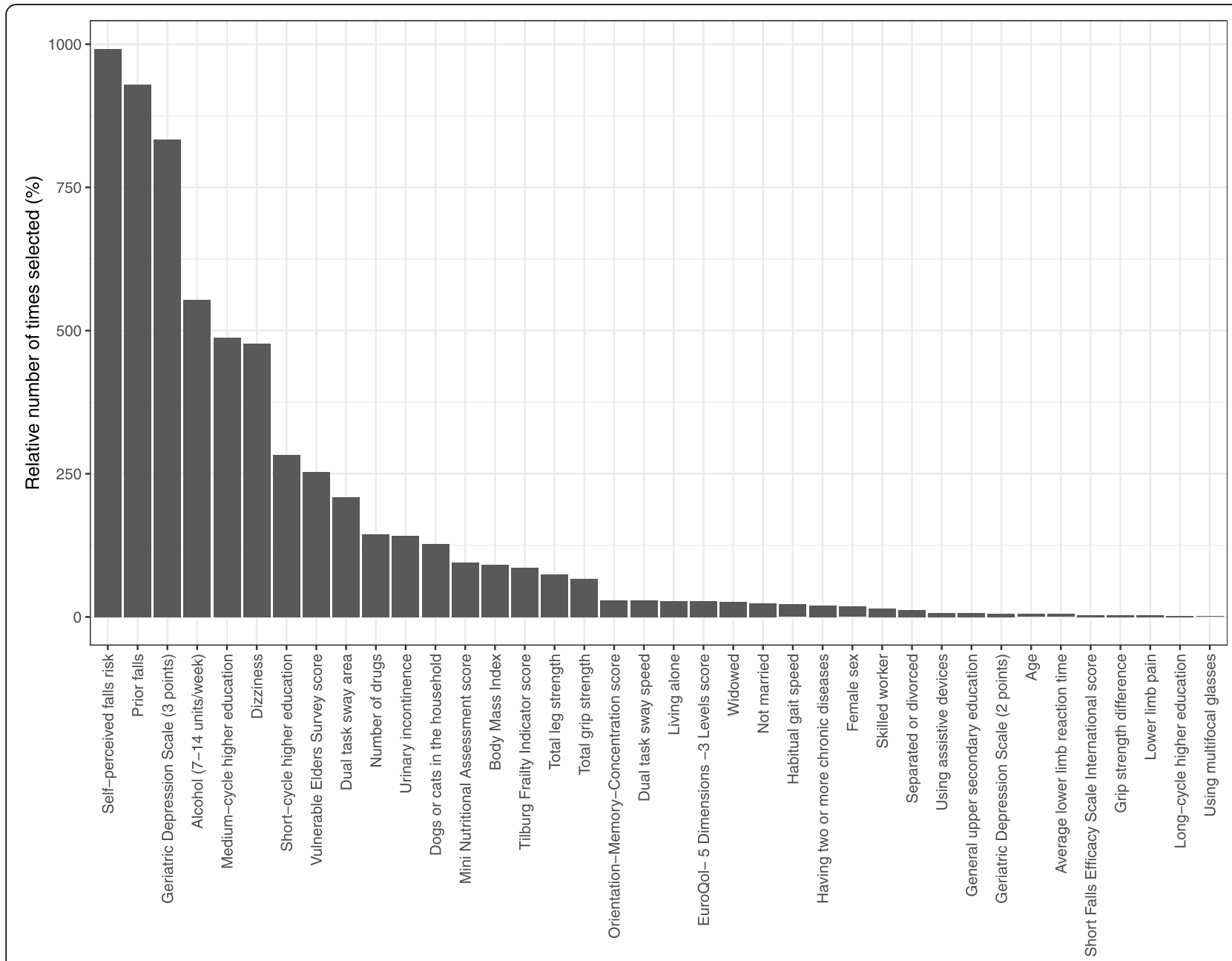

Fig. 2 Predictors most frequently selected for prediction models in 1000 bootstraps

implementable due to their simplicity. Regarding time consumption, our model included the Vulnerable Elders Survey 13 [31], taking five minutes to administer [40], and nine questions giving a completion time of approximately $15 \mathrm{~min}$. With current population prospects, it is highly relevant to identify fallers among older adults before they develop physical impairments that further increase their falls risk. However, our falls prediction model needs external validation before recommendation for clinical practice.

\section{Implications for research}

Since falls are multifactorial, they are also complex to predict. Hence, after externally validating our model, updating it with other predictors may further optimize performance. Other modeling studies have found static balance measures [14, 15] and comorbidities [11, 13, 15] to predict falls. We measured the latter but did not include this in the modeling process due to issues with implementability as diagnoses were not available for data collectors within our setting. Measures of executive and global cognitive function have also gained increased attention in falls research [41, 42]. Our study used a simple test for global cognitive function, OrientationMemory-Concentration test, and executive function using dual tasking during balance. Similar to lower limb strength, none of these predicted falls, which could be due to the tests not differentiating well between fallers and non-fallers. After we commenced the study, recommendations for tests examining the interactions between cognition and motor function were published [43]. Here, the Montréal Cognitive Assessment [44], Trail Making Test [45], and dual-task gait measures were highlighted [43]. These tests may be more challenging for wellfunctioning community-dwellers, which could potentially increase the predictive performance of a model. Lastly, physical activity measures $[11,16]$ have been used in models, and accelerometry measures have shown 
Table 3 Final prediction model on fall rate and performance measures

\begin{tabular}{ll}
\hline Sample size (n) & 241 \\
Number of events (falls) & 178 \\
Intercept and predictors & Beta coefficient \\
Intercept & -6.89417948 \\
Short-cycle higher education (completed) & 0.14752490 \\
Medium-cycle higher education & 0.22365429 \\
Occasional dizziness (present) & 0.17787573 \\
Weekly alcohol consumption: 7-14 units (yes) & 0.28918905 \\
Fallen within the last year (yes) & 0.50322396 \\
Self-perceived falls risk (will fall) & 0.74611041 \\
VES score & 0.01768701 \\
3 points on GDS & 1.21255321 \\
Performance & \\
Apparent mean squared error & 1.11 \\
Bootstrapped mean squared error (95\% Cl) & $1.92(0.81-5.10)$ \\
Apparent mean absolute error & 0.80 \\
Bootstrapped mean absolute error (95\% Cl) & 0.88 (0.71-1.16) \\
\hline
\end{tabular}

Notes: VES score Vulnerable Elders Survey 13 score, GDS Geriatric Depression Scale with 4 items, $\mathrm{kg}$ kilogram, $\mathrm{Cl}$ Confidence interval

promising results in falls prediction [46]. Future studies may consider updating our model using these predictors.

\section{Strengths and limitations}

The study has a number of strengths. Missing data were sparse in the sample, the design ensured blinding within the study, and the follow-up period was long, thereby increasing chances of falls occurring. We used current recommendations on falls definitions and recording methods and reported the study transparently [20, 22]. There were also some limitations present. Half of the participants were not consecutively sampled, and the recruitment rate was low. This may have introduced selection and non-response bias that could influence predictor-outcome relationships and predictive performance when predicting falls in everyday clinical practice. This may explain the increase in MAE when performing internal validation. Furthermore, we collected predictor data within a week rather than at one point in time. This choice was a practical solution to collect data on as many predictors as possible without compromising recruitment. Predictive performance may have been influenced only to a minor degree since none of the participants fell during the first week after enrolment (data not shown), and questionnaire predictors were known to be stable over time [34, 47-50]. Furthermore, the final model was questionnaire-based, and hence predictors could be available at the time of predicting.
Another limitation of the prediction model was the VES not being validated in Danish. Regarding univariate analyses, increasing age and high educational level were associated with a lower and higher falls rate, respectively. These findings are in contrast to other studies, where increasing age is associated with falls, and higher educational level is not [4]. In terms of age, this surprising finding could be due to survival bias. However, we performed a subsequent comparison between the ones dying during follow-up ( $n=3$, median age (IQR): 81 ( 82 , $83)$ years) and not $(n=238$, median age (IQR): $82(80$, $86)$ years) and found no statistically significant age difference $(p=0.89)$. Therefore, we do not believe survival bias to be of relevance in this study. However, the univariate analysis may explain this association since no adjustments for confounders were made, along with age not being included in the final model. The latter indicates that age was found less predictive of falls in the presence of other candidate predictors in this study. Regarding educational level, a comparison was made across its categories in terms of length of follow-up time, and no significant differences were found $(p=0.85)$. These findings may be due to selection bias since the same levels of education were included in the final prediction model. Overall, the results need to be confirmed in external validation studies to assess transportability to other populations.

\section{Conclusion}

We developed a falls prediction model applicable to well-functioning community-dwelling older adults. The model consists of a time-efficient self-reported questionnaire that could add fallers' early detection while being suitable for everyday clinical practice. The imprecision of the model was less than one fall. The results are promising, and we recommend external validation.

\section{Abbreviations}

PHV: Preventive home visits; SAC: Senior activity centers; MAE: Mean absolute error; IQR: Interquartile range; Cl: Confidence interval; IRR: Incidence rate ratio; TRIPOD: Transparent Reporting of a multivariable prediction model for Individual Prognosis Or Diagnosis; STROBE: Strengthening the Reporting of OBservational studies in Epidemiology; GDS4: Geriatric Depression Scale with four items; VES: Vulnerable Elders Survey 13; TFI: Tilburg Frailty Indicator; MNA: Mini Nutritional Assessment

\section{Supplementary Information}

The online version contains supplementary material available at https://doi. org/10.1186/s12877-021-02346-z.

Additional file 1. Data supplements. The data supplement provides appendices $1-4$ as referenced in the manuscript text.

\section{Acknowledgments}

We would like to express our gratitude to the study participants for dedicating their time and effort to the study. Also, we thank our research secretaries Anne Møller Rishøj and Christel Overgaard for handling fall calendars and telephone validation of falls. Furthermore, we would like to 
thank Hjørring Municipality for assisting with the recruitment and collection of baseline data. Finally, we would like to thank A.P. Møller Lægefonden for funding the hardware for this study.

\section{Authors' contributions}

Conceptualization, GVG, MGJ, JR, TM, and SA; Methodology, GVG, MGJ, JR, TM, SA, and LHJ; Software, GVG, MGJ, LHJ; Validation, GVG, SA; Formal analysis, GVG, LHJ; Investigation, GVG; Resources, SA, MGJ, GVG; Data curation, GVG, LHJ; Writing - Original Draft, GVG; Writing - Review \& Editing, GVG, MGJ, JR, TM, SA, and LHJ; Visualization, GVG; Supervision, MGJ, JR, TM, and SA; Project Administration, GVG; Funding Acquisition, SA and GVG. The authors read and approved the final manuscript.

\section{Funding}

This work was supported by A.P. Møller Lægefonden (grant number 18-L0377) funding the hardware for measuring balance, strength, weight, and reaction time. The funder had no role in the design, conduct, analysis, or reporting of the study. Otherwise, our host institutions funded this study.

\section{Availability of data and materials}

The dataset generated and analyzed during the current study is not publicly available due to the sensitivity of personal information but is available from the corresponding author on reasonable request.

\section{Declarations}

\section{Ethics approval and consent to participate}

The study was conducted in accordance with Danish Legislation and the Declaration of Helsinki. The North Denmark Region Committee on Health Research Ethics was contacted before study initiation, and the study was granted an exemption from requiring ethics approval. The study was registered at the Danish Data Protection Agency (2018-82). Enrolment required written informed consent preceded by both oral and written information on the study.

\section{Consent for publication}

Not applicable.

\section{Competing interests}

MGJ holds shares in the company Fysiometer which produced the hard- and software used for measuring balance, reaction time, weight, along with gripand lower limb strength. All other authors declare that they have no competing interests.

\section{Author details}

'Department of Geriatric Medicine, Aalborg University Hospital, Hobrovej 18-22, Gl. Roed Bygn. 6, 9000 Aalborg, Denmark. ²Department of Clinical Medicine, Aalborg University, Soendre Skovvej 15, 9000 Aalborg, Denmark. ${ }^{3}$ Department of Geriatric Medicine, Odense University Hospital, Sdr. Boulevard 29, 5000 Odense, Denmark. ${ }^{4}$ Department of Clinical Research, University of Southern Denmark, Winsloewparken 19, 5000 Odense, Denmark. ${ }^{5}$ Department of Healthcare for Older People, Nottingham University Hospitals NHS Trust, Derby Road, Nottingham, Nottinghamshire NG7 2UH, UK. ${ }^{6}$ Department of Hematology, Aalborg University Hospital, Moelleparkvej 4, Aalborg, Denmark.

\section{Received: 23 March 2021 Accepted: 7 June 2021}

\section{Published online: 30 June 2021}

\section{References}

1. United Nations, Department of Economic and Social Affairs PD. World population prospects 2019: Highlights (ST/ESA/SER.A/423). 2019.

2. Rubenstein LZ. Falls in older people: Epidemiology, risk factors and strategies for prevention. Age Ageing. 2006;35(Suppl 2):ii35-41.

3. Cummings-Vaughn LA, Gammack JK. Falls, Osteoporosis, and Hip Fractures. Med Clin North Am. 2011;95:495-506.

4. Deandrea S, Lucenteforte E, Bravi F, Foschi R, La Vecchia C, Negri E. Risk factors for falls in community-dwelling older people: a systematic review and meta-analysis. Epidemiology. 2010;21(5):658-68. https://doi.org/10.1097/ EDE.0b013e3181e89905.
5. Scuffham P, Chaplin S, Legood R. Incidence and costs of unintentional falls in older people in the United Kingdom. J Epidemiol Community Health. 2003;57(9):740-4. https://doi.org/10.1136/jech.57.9.740.

6. Panel on Prevention of Falls in Older People American Geriatrics Society and British Geriatrics Society. Summary of the updated American Geriatrics Society/British Geriatrics Society clinical practice guideline for prevention of falls in older persons. J Am Geriatr Soc. 2011;59(1):148-57.

7. Inouye SK, Studenski S, Tinetti M, Kuchel G. Geriatric syndromes: clinical, research and policy implications of a Core geriatric concept in older adults related to primary and secondary prevention. J Am Geriatr Soc. 2007;55(5): 780-91. https://doi.org/10.1111/j.1532-5415.2007.01156.X.

8. Moons KGM, Royston P, Vergouwe Y, Grobbee DE, Altman DG. Prognosis and prognostic research: What, why, and how? BMJ. 2009;338:1317-20.

9. Steyerberg EW, Moons KG, van der Windt DA, Hayden JA, Perel P, Schroter S, Riley RD, Hemingway H, Altman DG, PROGRESS Group. Prognosis research strategy (PROGRESS) 3: prognostic model research. PLoS Med. 2013;10(2): e1001381. https://doi.org/10.1371/journal.pmed.1001381. PMID: 23393430; PMCID: PMC3564751.

10. Gade GV, Jørgensen MG, Ryg J, Riis J, Thomsen K, Masud T, et al. Predicting falls in community-dwelling older adults: a systematic review of prognostic models. BMJ Open. 2021:11:e044170.

11. De Vries OJ, Peeters GMEE, Lips P, Deeg DJH. Does frailty predict increased risk of falls and fractures? A prospective population-based study. Osteoporos Int. 2013;24(9):2397-403. https://doi.org/10.1007/s00198-013-23 03-z.

12. Pluijm SMF, Smit JH, Tromp EAM, Stel VS, Deeg DJH, Bouter LM, et al. A risk profile for identifying community-dwelling elderly with a high risk of recurrent falling: results of a 3-year prospective study. Osteoporos Int. 2006; 17(3):417-25. https://doi.org/10.1007/s00198-005-0002-0.

13. Tromp AM, Pluijm SMF, Smit JH, Deeg DJ. JH, Bouter LM, Lips P, et al. Fallrisk screening test: a prospective study on predictors for falls in communitydwelling elderly. J Clin Epidemiol 2001;54(8):837-844, DOl: https://doi.org/1 0.1016/S0895-4356(01)00349-3.

14. Maki BE, Holliday PJ, Topper AK. A prospective study of postural balance and risk of falling in an ambulatory and independent elderly population. J Gerontol. 1994:49(2):M72-84. https://doi.org/10.1093/geronj/49.2.M72

15. Gadkaree SK, Sun DQ, Huang J, Varadhan R, Agrawal Y. Comparison of simple vs. performance-based fall prediction models: data from the National Health and Aging Trends Study. Gerontol Geriatr Med. 2015;1: 2333721415584850. https://doi.org/10.1177/2333721415584850. PMID: 26702410; PMCID: PMC4686273.

16. Stel VS, Pluijm SMF, Deeg DJH, Smit JH, Bouter LM, Lips P. A classification tree for predicting recurrent falling in community-dwelling older persons. J Am Geriatr Soc. 2003;51(10):1356-64. https://doi.org/10.1046/j.1532-5415.2 003.51452.x

17. Mi A, Ruiz J, Valldosera E, Gonzalo N, Ng T, Yuste A, et al. A two-question tool to assess the risk of repeated falls in the elderly. PLoS One. 2017;12(5): e0176703.

18. Royston P, Moons KGM, Altman DG, Vergouwe Y. Prognosis and prognostic research: developing a prognostic model. BMJ. 2009;338(7707):1373-7.

19. Sørensen G, Jorgensen M, Ryg J, Masud T, Andersen S. Development of a Multivariable Prognostic PREdiction Model for 1-year Risk of FALLing in Community-dwelling Older Adults in a Non-clinical Setting (PROTOCOL) [Internet]. [cited 2020 Jul 7]. Available from: https://clinicaltrials.gov/ct2/ show/NCT03608709.

20. Collins GS, Reitsma JB, Altman DG, Moons K. Transparent reporting of a multivariable prediction model for individual prognosis or diagnosis (TRIPOD): the TRIPOD statement. BMC Med. 2015;13(1):1. https://doi.org/1 0.1186/s12916-014-0241-z.

21. von Elm E, Altman DG, Egger M, Pocock SJ, Gøtzsche PC, Vandenbroucke JP, et al. The strengthening the reporting of observational studies in epidemiology (STROBE) statement: guidelines for reporting observational studies. Lancet. 2007;370(9596): 1453-7. https://doi.org/10.1016/S0140-6736(07)61602-X.

22. Lamb SE, Jørstad-Stein EC, Hauer K, Becker C. Development of a common outcome data set for fall injury prevention trials: the prevention of falls network Europe consensus. J Am Geriatr Soc. 2005;53(9):1618-22. https:// doi.org/10.1111/j.1532-5415.2005.53455.x.

23. Sørensen GV, Jørgensen MG, Ryg J, Masud T, Andersen S. Development feasibility, acceptability, and adjustment of a portable, multifactorial falls risk test battery for community-dwelling older adults. Cogent Med. 2019;6(1): 1674099. https://doi.org/10.1080/2331205X.2019.1674099. 
24. Blomkvist AW, Andersen S, de Bruin E, Jorgensen MG. Unilateral lower limb strength assessed using the Nintendo Wii balance board: a simple and reliable method. Aging Clin Exp Res. 2017;29(5):1013-20. https://doi.org/10.1 007/s40520-016-0692-5.

25. Jorgensen MG, Paramanathan S, Ryg J, Masud T, Andersen S, Rubenstein L, et al. Novel use of the Nintendo Wii board as a measure of reaction time: a study of reproducibility in older and younger adults. BMC Geriatr. 2015; 15(1):80. https://doi.org/10.1186/s12877-015-0080-6.

26. Blomkvist AW, Andersen S, De Bruin ED, Jorgensen MG. Isometric hand grip strength measured by the Nintendo Wii balance board - a reliable new method rehabilitation, physical therapy and occupational health. BMC Musculoskelet Disord. 2016;17(1):1-7.

27. Jørgensen MG, Laessoe U, Hendriksen C, Nielsen OBF, Aagaard P, Jorgensen $M G$, et al. Intrarater reproducibility and validity of Nintendo Wii balance testing in community-dwelling older adults. J Aging Phys Act. 2014;22(2): 269-75. https://doi.org/10.1123/japa.2012-0310.

28. Quach L, Galica AM, Jones RN, Procter-Gray E. The non-linear relationship between gait speed and falls:the mobilize Boston study. J Am Geriatr Soc. 2012;59(6):1069-73.

29. Andreasen J, Sørensen EE, Gobbens RJJ, Lund H, Aadahl M. Danish version of the Tilburg frailty Indicator - translation, cross-cultural adaption and validity pretest by cognitive interviewing. Arch Gerontol Geriatr. 2014;59(1): 32-8. https://doi.org/10.1016/j.archger.2014.02.007.

30. Rubenstein LZ, Harker JO, Salvà A, Guigoz Y, Vellas B. Screening for undernutrition in geriatric practice : developing the short form mini nutritional assessment (MNA-SF). J Gerontol. 2001;56(6):366-72.

31. Saliba D, Elliott M, Rubenstein LZ, Solomon DH, Young RT, Kamberg CJ, et al. The vulnerable Elders survey: a tool for identifying vulnerable older people in the community. J Am Geriatr Soc. 2001;49(12):1691-9. https://doi. org/10.1046/j.1532-5415.2001.49281.x.

32. Kempen GIJM, Yardley L, Van Haastregt JCM, Zijlstra GAR, Beyer N, Hauer K, et al. The short FES-l: a shortened version of the falls efficacy scaleinternational to assess fear of falling. Age Ageing. 2008:37(1):45-50. https:// doi.org/10.1093/ageing/afm157.

33. D'Ath P, Katona P, Mullan E, Evans S, Katona C. Screening, detection and Management of Depression in elderly primary care attenders. I: the acceptability and performance of the 15 item geriatric depression scale (GDS15) and the development of short versions. Fam Pr. 1994;11(3):260-6. https://doi.org/10.1093/fampra/11.3.260.

34. Wade DT, Vergis E. The short orientation-memory-concentration test: a study of its reliability and validity. Clin Rehabil. 1999;13(2):164-70. https:// doi.org/10.1191/026921599673848768.

35. Ambler G, Omar RZ, Royston P. A comparison of imputation techniques for handling missing predictor values in a risk model with a binary outcome. Stat Methods Med Res. 2007;16(3):277-98. https://doi.org/10.1177/09622802 06074466

36. Altman DG, Royston P. The cost of dichotomising continuous variables. BMJ. 2006;332(7549):1080. https://doi.org/10.1136/bmj.332.7549.1080. PMID: 16675816; PMCID: PMC1458573.

37. Friedman J, Hastie T, Tibshirani R. Regularization paths for generalized linear models via coordinate descent. J Stat Softw. 2010;33(1):1-22.

38. Moreland JD, Richardson JA, Goldsmith CH, Clase CM. Muscle weakness and falls in older adults: A systematic review and meta-analysis. J Am Geriatr Soc. 2004,52:1121-9.

39. Eika F, Blomkvist AW, Rahbek MT, Eikhof KD, Hansen MD, Søndergaard M, et al. Reference data on hand grip and lower limb strength using the Nintendo Wii balance board: a cross-sectional study of 354 subjects from 20 to 99 years of age. BMC Musculoskelet Disord. 2019;20(1):21. https://doi. org/10.1186/s12891-019-2405-7.

40. Decoster L, Van Puyvelde K, Mohile S, Wedding U, Basso U, Colloca G, et al. Screening tools for multidimensional health problems warranting a geriatric assessment in older cancer patients: An update on SIOG recommendations. Ann Oncol. 2015;26:288-300.

41. Muir-Hunter SW, Wittwer JE. Dual-task testing to predict falls in communitydwelling older adults: a systematic review. Physiotherapy. 2016;102(1):29-40. https://doi.org/10.1016/j.physio.2015.04.011.

42. Muir SW, Gopaul K, Montero Odasso MM. The role of cognitive impairment in fall risk among older adults: a systematic review and meta-analysis. Age Ageing. 2012;41(3):299-308. https://doi.org/10.1093/ageing/afs012.

43. Montero-Odasso M, Almeida QJ, Bherer L, Burhan AM, Camicioli R, Doyon J, et al. Consensus on Shared Measures of Mobility and Cognition: From the
Canadian Consortium on Neurodegeneration in Aging (CCNA). J Gerontol Ser A. 2018;XX((XX):1-13.

44. Nasreddine ZS, Phillips NA, Bédirian V, Charbonneau S, Whitehead V, Collin I, et al. The Montreal cognitive assessment, MoCA: a brief screening tool for mild cognitive impairment. J Am Geriatr Soc. 2005;53(4):695-9. https://doi. org/10.1111/j.1532-5415.2005.53221.x.

45. Vazzana R, Bandinelli S, Lauretani F, Volpato S, Lauretani F, Di lorio A, et al. Trail making test predicts physical impairment and mortality in older persons. J Am Geriatr Soc. 2010;58(4):719-23. https://doi.org/10.1111/j.15325415.2010.02780.x.

46. Van Schooten KS, Pijnappels M, Rispens SM, Elders PJM, Lips P, Daffertshofer A, et al. Daily-life gait quality as predictor of falls in older people: a 1-year prospective cohort study. PLoS One. 2016;11(7):1-13.

47. Davis JC, Best JR, Dian L, Khan KM, Hsu CL, Chan W, et al. Are the EQ-5D-3L and the ICECAP-O responsive among older adults with impaired mobility? Evidence from the Vancouver falls prevention cohort study. Qual Life Res. 2017:26(3):737-47. https://doi.org/10.1007/s11136-016-1487-z.

48. Yardley L, Beyer N, Hauer K, Kempen G, Piot-Ziegler C, Todd C. Development and initial validation of the falls efficacy scale-international (FES-I). Age Ageing. 2005;34(6):614-9. https://doi.org/10.1093/ageing/afi196.

49. Keller HH, McKenzie JD, Goy RE. Construct validation and test-retest reliability of the seniors in the community: risk evaluation for eating and nutrition questionnaire. J Gerontol Ser A Biol Sci Med Sci. 2001;56(9):552-8.

50. Sutton JL, Gould RL, Daley S, Coulson MC, Ward EV, Butler AM, Nunn SP, Howard RJ. Psychometric properties of multicomponent tools designed to assess frailty in older adults: a systematic review. BMC Geriatr. 2016;16:55. https://doi.org/10.1186/s12877-016-0225-2. PMID: 26927924; PMCID: PMC4772336.

\section{Publisher's Note}

Springer Nature remains neutral with regard to jurisdictional claims in published maps and institutional affiliations.
Ready to submit your research? Choose BMC and benefit from:
- fast, convenient online submission
- thorough peer review by experienced researchers in your field
- rapid publication on acceptance
- support for research data, including large and complex data types
- gold Open Access which fosters wider collaboration and increased citations
- maximum visibility for your research: over $100 \mathrm{M}$ website views per year
At BMC, research is always in progress.
Learn more biomedcentral.com/submissions 